\title{
Chiral bosons and improper constraints
}

\author{
F. P. Devecchi and H. O. Girotti \\ Instituto de Física, Universidade Federal do Rio Grande do Sul, Caixa Postal 15051, 91501-970 Porto Alegre, RS, Brazil
}

(Received 29 September 1993)

\begin{abstract}
We argue that a consistent quantization of the Floreanini-Jackiw model, as a constrained system, should start by recognizing the improper nature of the constraints. Then, each boundary condition defines a problem which must be treated separately. The model is settled on a compact domain which allows for a discrete formulation of the dynamics; thus, avoiding the mixing of local with collective coordinates. For periodic boundary conditions the model turns out to be a gauge theory whose gauge invariant sector contains only chiral excitations. For antiperiodic boundary conditions, the model is a second-class theory where the excitations are also chiral. In both cases, the equal-time algebra of the quantum energy-momentum densities is a Virasoro algebra. The Poincaré symmetry holds for the finite as well as for the infinite domain.
\end{abstract}

PACS number(s): 11.30.Qc, 11.10.Ef, 11.30.Ly

Self dual fields, also known as chiral bosons, are of interest due to their relevance in the heterotic string [1] and in the quantum Hall effect [2]. The quantization of these objects is beset with difficulties. Indeed, the Lorentz invariant model in Refs. [3,4], based on the idea that the chiral condition could be implemented through a linear constraint, does not exhibit physical excitations [5]. On the other hand, the canonical quantization of Siegel's Lagrangian [6] is afflicted by an anomaly which is to be eliminated by the addition of a Wess-Zumino term. It turns out, then, that the resulting theory does not describe pure chiral bosons but rather their coupling to gravity [7].

Of particular interest is the $(1+1)$-dimensional model put forward by Floreanini and Jackiw (FJ) [8,9], whose dynamics is described by the noncovariant Lagrangian density

$$
\mathcal{L}=\frac{1}{2}\left(\partial_{0} \phi\right)\left(\partial_{1} \phi\right)-\frac{1}{2}\left(\partial_{1} \phi\right)\left(\partial_{1} \phi\right)
$$

where $x \equiv\left(x^{0}, x^{1}\right)$ and $\phi \equiv \phi(x)$ is a real scalar field whose canonically conjugate momentum will be denoted as $\pi(x)$. This model was quantized through the Diracbrackets procedure [10] by one of us (H.O.G.) in collaboration with Costa $[11,12]$. It possesses a nondenumerable set of constraints,

$$
\gamma\left(x^{0}, x^{1}\right) \equiv \pi\left(x^{0}, x^{1}\right)-\frac{1}{2} \partial_{1} \phi\left(x^{0}, x^{1}\right) \approx 0,
$$

while the canonical Hamiltonian is $H_{c}=$ $\frac{1}{2} \int_{-\infty}^{+\infty} d x^{1}\left(\partial_{1} \phi\right)\left(\partial_{1} \phi\right)$. One readily verifies that the Poisson brackets of the constraints define a matrix $Q$ whose inverse is not unique:

$$
Q^{-1}\left(x^{0} ; x^{1}, y^{1}\right)=-\frac{1}{2} \epsilon\left(x^{1}-y^{1}\right)+\zeta\left(x^{0}\right) .
$$

Here, $\epsilon\left(x^{1}\right)$ denotes the sign function. In Ref. [11] the arbitrary function $\zeta$ was set to zero. By abstracting the equal-time commutators from the corresponding Dirac brackets (Dirac-brackets quantization procedure) the basic equal-time commutation relations were then found [11]. In particular, we quote

$$
\left[\hat{\phi}\left(x^{1}\right), \hat{\phi}\left(y^{1}\right)\right]=-\frac{i}{2} \epsilon\left(x^{1}-y^{1}\right)
$$

where the carets denote operators. One can check that the chiral field configuration

$$
\begin{aligned}
\hat{\phi}\left(x^{0}, x^{1}\right)= & \frac{1}{\sqrt{2 \pi}} \int_{0}^{\infty} d k^{1} \frac{1}{\sqrt{k^{1}}}\left[\hat{\Lambda}\left(k^{1}\right) e^{-i k^{1}\left(x^{0}+x^{1}\right)}\right. \\
& \left.+\hat{\Lambda}^{\dagger}\left(k^{1}\right) e^{i k^{1}\left(x^{0}+x^{1}\right)}\right]
\end{aligned}
$$

with $\hat{\Lambda}\left(k^{1}\right)$ and $\hat{\Lambda}^{\dagger}\left(k^{1}\right)$ destruction and creation operators, respectively, solves the Heisenberg equation of motion arising from $\hat{H}_{c}$ and the equal-time commutation relation (4).

The presence of the arbitrary function $\zeta$ in (3) indicates that the solution (5) is not unique [13] and, therefore, cast doubts on whether all physical excitations of the FJ model are in fact chiral. To elucidate this point is the main purpose of this work.

We start by recognizing that the constraints $\gamma$ are improper [14-16]. Indeed, from (2) it follows that

$$
\begin{aligned}
\delta \gamma[\eta]= & \int_{-\infty}^{+\infty} d x^{1}\left(\eta\left(x^{1}\right) \delta \pi\left(x^{1}\right)+\frac{1}{2} \partial_{1} \eta\left(x^{1}\right) \delta \phi\left(x^{1}\right)\right) \\
& -\frac{1}{2}[\eta(\infty) \delta \phi(\infty)-\eta(-\infty) \delta \phi(-\infty)]
\end{aligned}
$$

where $\eta\left(x^{1}\right)$ is any function in the space dual to the space of constraints $\gamma\left(x^{1}\right)$ and $\gamma[\eta] \equiv \int_{-\infty}^{+\infty} d x^{1} \eta\left(x^{1}\right) \gamma\left(x^{1}\right)$. The presence of the surface term in (6) confirms the improper nature of the constraints (2). In order for the total Hamiltonian [10] to be a proper generator of time transformations, one must require the vanishing of this surface term. Hence, the construction of the dual space depends on the boundary conditions of $\delta \phi\left(x^{1}\right)$. For instance, $\delta \phi(\infty)=\delta \phi(-\infty)$ demands $\eta(\infty)=\eta(-\infty)$, while $\delta \phi(\infty)=-\delta \phi(-\infty)$ demands $\eta(\infty)=-\eta(-\infty)$.

Thus, each set of histories $\{\phi(x)\}$ verifying a certain boundary condition defines a problem which must be treated separately. In this work the FJ model is settled on a compact domain $\left(-R \leq x^{1} \leq+R\right)$ and then 
quantized under periodic and antiperiodic boundary conditions. As we shall see, to each boundary condition corresponds a different constraint structure; in particular, only periodic boundary conditions allow for first-class constraints. The quantum energy-momentum densities are constructed and their equal-time algebra is investigated. A set of Poincaré charges is built and the limit $R \rightarrow \infty$ is analyzed in both cases.

Let $\left\{\phi\left(x^{0}, x^{1}\right) \mid \phi\left(x^{0},+R\right)=\phi\left(x^{0},-R\right)\right\}$ be the set of periodic histories and let $\phi\left(x^{0}, x^{1}\right)$ be any history in this set. One readily verifies that, in this case, the action

$$
S[\phi]=\int_{-R}^{+R} d x^{1}\left[\frac{1}{2}\left(\partial_{0} \phi\right)\left(\partial_{1} \phi\right)-\frac{1}{2}\left(\partial_{1} \phi\right)\left(\partial_{1} \phi\right)\right]
$$

is invariant under the transformation

$$
\phi\left(x^{0}, x^{1}\right) \longrightarrow \phi\left(x^{0}, x^{1}\right)+f\left(x^{0}\right)
$$

which preserves the boundary conditions [14]. This transformation, which is neither local nor global, must be generated by a first-class constraint. Such a generator, $\Gamma\left(x^{0}\right)$, is an infinite combination of the constraints (2) and reads

$$
\Gamma\left(x^{0}\right)=\int_{-R}^{+R} d x^{1} \pi\left(x^{0}, x^{1}\right) \approx 0 .
$$

Of course, the invariance of the action under the transformation (8) is responsible for the lack of uniqueness present in (3). This "gauge freedom" cannot be fixed by means of local gauge conditions. Alternatively, one may try fixing by means of an integrated condition. When quantizing the theory along these lines one faces the problem of computing Dirac brackets in which local field variables are mixed with collective ones [17].

To free ourselves from the above drawbacks, we shall quantize the FJ model by taking advantage of the compactness of the domain. This, together with the boundary conditions under analysis, allows for a discrete formulation of the theory in terms of the real Fourier coefficients $a_{0}\left(x^{0}\right), a_{n}\left(x^{0}\right)$ and $b_{n}\left(x^{0}\right)$ entering in the decomposition of the real field $\phi[18]$ :

$$
\begin{aligned}
\phi\left(x^{0}, x^{1}\right)= & \frac{1}{2 R} a_{0}\left(x^{0}\right) \\
& +\frac{1}{2 R} \sum_{n>0}\left\{\left[a_{n}\left(x^{0}\right)+i b_{n}\left(x^{0}\right)\right] e^{\frac{i n \pi}{R} x^{1}}\right. \\
& \left.+\left[a_{n}\left(x^{0}\right)-i b_{n}\left(x^{0}\right)\right] e^{-\frac{i n \pi}{R} x^{1}}\right\}
\end{aligned}
$$

where $n \in \mathcal{Z}$. By starting from (1) and (10) and going through the usual canonical analysis [10] one finds that the system possesses a primary first-class constraint $p_{a_{0}} \approx 0$, a set of primary second-class constraints $(n>0)$

$$
\begin{aligned}
& \Gamma_{P_{n}}^{+} \equiv p_{a_{n}}+\frac{\omega_{n}}{2 R} b_{n} \approx 0 \\
& \Gamma_{P_{n}}^{-} \equiv p_{b_{n}}-\frac{\omega_{n}}{2 R} a_{n} \approx 0
\end{aligned}
$$

while the canonical Hamiltonian $H_{c}^{P}$ reads

$$
H_{c}^{P}=\frac{1}{2 R} \sum_{n>0} \omega_{n}^{2}\left(a_{n}^{2}+b_{n}^{2}\right)
$$

Here, $\omega_{n} \equiv n \pi / R$ whereas $p_{a_{n}}$ and $p_{b_{n}}$ are the momenta canonically conjugate to $a_{n}$ and $b_{n}$, respectively. It is easy to see that there are no secondary constraints. The first-class constraint $p_{a_{0}} \approx 0$ is the discrete counterpart of (9). It generates gauge transformations that only affect $a_{0}$, i.e., the collective part of $\phi\left(x^{0}, x^{1}\right)$. All phase-space coordinates for $n>0$ are, then, gauge invariant quantities. However, unlike the continuous case, the gauge freedom can now be suppressed by means of a subsidiary condition and the system quantized, afterward, via the Dirac-brackets procedure. Without losing generality we assume for the gauge fixing condition the functional form: $a_{0}+\xi\left(a_{n}, b_{n}, p_{a_{n}}, p_{b_{n}}\right) \approx 0$. For the $n>0$ sector the basic nonvanishing commutator turns out to be

$$
\left[\hat{a}_{n}, \hat{p}_{a_{m}}\right]=\frac{i}{2} \delta_{n, m}
$$

As far as the commutators involving $\hat{a}_{0}$ and/or $\hat{p}_{a_{0}}$ are concerned, we mention that they can be explicitly computed only after specifying the gauge function $\xi$. The Hamiltonian $H_{c}^{P}$ can be promoted to the quantum level straightforwardly because is not afflicted by ordering ambiguities. Then, the solving of the Heisenberg equations of motion for the independent phase-space variables yields

$$
\hat{a}_{n}\left(x^{0}\right)=\sqrt{\frac{\pi}{2 \omega_{n}}} \hat{\Lambda}_{n} e^{-i \omega_{n} x^{0}}+\sqrt{\frac{\pi}{2 \omega_{n}}} \hat{\Lambda}_{n}^{\dagger} e^{i \omega_{n} x^{0}}
$$

The operators $\hat{\Lambda}_{n}$ and $\hat{\Lambda}_{n}^{\dagger}$ are destruction and creation operators, respectively. Thus, the space of states is of positive definite norm although the vacuum $(|0\rangle)$ is not unique. Indeed, (10) can be cast as

$$
\begin{aligned}
\hat{\phi}\left(x^{0}, x^{1}\right)= & -\frac{\hat{\xi}}{2 R} \\
& +\frac{1}{\sqrt{2 \pi}}\left(\frac{\pi}{R}\right) \sum_{n>0} \frac{1}{\sqrt{\omega_{n}}}\left[\hat{\Lambda}_{n} e^{-i \omega_{n}\left(x^{0}+x^{1}\right)}\right. \\
& \left.+\hat{\Lambda}_{n}^{\dagger} e^{i \omega_{n}\left(x^{0}+x^{1}\right)}\right]
\end{aligned}
$$

implying that

$$
\left\langle 0\left|\hat{\phi}\left(x^{0}, x^{1}\right)\right| 0\right\rangle=-\frac{1}{2 R}\langle 0|\hat{\xi}(0)| 0\rangle \neq 0,
$$

where we have used $\hat{\Lambda}_{n}|0\rangle=0$ and the fact that all vacua are translationally invariant.

The collective mode in (15) is neither gauge invariant nor chiral and is responsible, as just observed, for the spontaneous breaking of the continuous symmetry $\phi \rightarrow \phi+$ const [see (1)]. That this remains true at the limit $R \rightarrow \infty$ follows from purely dimensional arguments. Nevertheless, a gauge invariant field operator $(\hat{\Phi})$ with vanishing vacuum expectation value can be naturally built within the discretized formulation:

$$
\hat{\Phi}\left(x^{0}, x^{1}\right) \equiv \hat{\phi}\left(x^{0}, x^{1}\right)+\frac{\hat{\xi}}{2 R} \text {. }
$$


Clearly, the field $\hat{\Phi}$ only describes chiral excitations. Furthermore, it obeys the equal-time commutation relation

$$
\begin{aligned}
& {\left[\hat{\Phi}\left(x^{0}, x^{1}\right), \hat{\Phi}\left(x^{0}, y^{1}\right)\right]} \\
& \quad=-\frac{i}{2}\left\{\frac{1}{i \pi} \sum_{n=-\infty}^{+\infty}\left(\frac{\pi}{R}\right) \frac{e^{\frac{i n \pi}{R}\left(x^{1}-y^{1}\right)}}{\frac{n \pi}{R}}\right\} .
\end{aligned}
$$

We investigate next the limit $R \rightarrow \infty$ for the results obtained within the discrete approach. In particular, the right-hand sides of (17) and (18) go, respectively, to

$$
\begin{gathered}
\hat{\Phi}\left(x^{0}, x^{1}\right)=\frac{1}{\sqrt{2 \pi}} \int_{0^{+}}^{\infty} d k^{1} \frac{1}{\sqrt{k^{1}}}\left[\hat{\Lambda}\left(k^{1}\right) e^{-i k^{1}\left(x^{0}+x^{1}\right)}\right. \\
\left.+\hat{\Lambda}^{\dagger}\left(k^{1}\right) e^{i k^{1}\left(x^{0}+x^{1}\right)}\right] \\
{\left[\hat{\Phi}\left(x^{0}, x^{1}\right), \hat{\Phi}\left(x^{0}, y^{1}\right)\right]=-\frac{i}{2} \epsilon\left(x^{1}-y^{1}\right)}
\end{gathered}
$$

where the linear momentum variable $k^{1}$ is the continuous version of the discrete variable $n \pi / R$, while $\hat{\Lambda}\left(k^{1}\right)$ and $\hat{\Lambda}^{\dagger}\left(k^{1}\right)$ are the limiting forms of the corresponding discrete destruction and creation operators. Then, the gauge invariant field $\hat{\Phi}$ remains chiral at the continuous limit and, moreover, (19) and (20) agree with (5) and (4), respectively. Hence, what was done in Ref. [11] by choosing arbitrarily $\zeta=0$, amounts to isolate, within the continuous framework, the gauge invariant piece of the operator $\hat{\phi}$.

As for the Poincaré invariance of the present formulation of the FJ model, we start by recalling that the classical energy-momentum tensor $\left(\Theta^{\mu \nu}\right)$, arising from the noncovariant Lagrangian density (1), satisfies $\partial_{\mu} \Theta^{\mu \nu}=0$ but is not symmetric. In fact, one finds that

$$
\begin{gathered}
\Theta^{00}=-\Theta^{01}=\Theta^{11}=\frac{1}{2}\left(\partial_{1} \phi\right)\left(\partial_{1} \phi\right), \\
\Theta^{10}=\frac{1}{2}\left(\partial_{0} \phi\right)\left(\partial_{0} \phi\right)-\left(\partial_{0} \phi\right)\left(\partial_{1} \phi\right) .
\end{gathered}
$$

The classical components of the energy-momentum tensor serve as a clue for establishing the form of the quantum densities $\hat{\Theta}^{\mu \nu}$ in terms of the basic fields [19]. A gauge invariant and symmetric quantum energy momentum tensor can be constructed by formally replacing $\phi$ by $\hat{\Phi}$ in (21):

$$
\hat{\Theta}^{00}=-\hat{\Theta}^{01}=-\hat{\Theta}^{10}=\hat{\Theta}^{11}=\frac{1}{2}:\left(\partial_{1} \hat{\Phi}\right)\left(\partial_{1} \hat{\Phi}\right):
$$

The normal ordering prescription introduced in (22) secures that $\left\langle 0\left|\hat{\Theta}^{\mu \nu}\right| 0\right\rangle=0$. We emphasize that the symmetric character of $\hat{\Theta}^{\mu \nu}$ is a consequence of the chiral nature of $\hat{\Phi}$.

The next step consists in investigating the equaltime commutation relations verified by the quantum energy-momentum densities. In view of (22), there is only one commutator of interest, namely, $\left[\hat{\Theta}^{00}\left(x^{0}, x^{1}\right), \hat{\Theta}^{00}\left(x^{0}, y^{1}\right)\right]$. After some calculations one arrives at

$$
\begin{aligned}
& {\left[\hat{\Theta}^{00}\left(x^{0}, x^{1}\right), \hat{\Theta}^{00}\left(x^{0}, y^{1}\right)\right]} \\
& \quad=i\left(\hat{\Theta}^{00}\left(x^{0}, x^{1}\right)+\hat{\Theta}^{00}\left(x^{0}, y^{1}\right)\right) \partial_{x^{1}} \delta\left(x^{1}-y^{1}\right) \\
& \quad-i \Delta\left(x^{1}, y^{1}\right)
\end{aligned}
$$

where

$$
\Delta\left(x^{1}, y^{1}\right)=\frac{1}{24 \pi} \partial_{x^{1}}^{3} \delta\left(x^{1}-y^{1}\right)+\frac{\pi}{24 R^{2}} \partial_{x^{1}} \delta\left(x^{1}-y^{1}\right)
$$

The algebra (23) is a Virasoro algebra, since the additional piece in the right-hand side of (24) $\left[\left(\pi / 24 R^{2}\right) \partial_{x^{1}} \delta\left(x^{1}-y^{1}\right)\right]$ is a trivial cocycle that can be absorbed in a constant redefinition of the energymomentum tensor.

We now show that the charges arising from $\Theta^{\mu \nu}$ are the generators of the Poincaré symmetry. Indeed, by integrating both sides of (23) over the domain of the variable $y^{1}$ one obtains

$$
\left[\hat{\Theta}^{00}\left(x^{0}, x^{1}\right), \hat{P}^{0}\right]=i \partial_{x^{1}} \hat{\Theta}^{00}\left(x^{0}, x^{1}\right),
$$

where $\hat{P^{0}} \equiv \int_{-R}^{+R} d x^{1} \hat{\Theta}^{00}\left(x^{0}, x^{1}\right)$ is the generator of translations in time. A subsequent $x^{1}$ integration in (25), including the factor $x^{1}$, gives

$$
\left[\hat{P^{0}}, \hat{M}\right]=i \hat{P^{0}}
$$

where $\hat{M} \equiv-x^{0} \hat{P}^{1}+\int_{-R}^{+R} d x^{1} x^{1} \hat{\Theta}^{00}\left(x^{0}, x^{1}\right)$ is the Lorentz boosts generator and $\hat{P}^{1}=-\hat{P}^{0}$ is the generator of spatial translations. What we have in (26) is, precisely, the contracted Poincaré algebra of Ref. [8]. This concludes our study of the FJ model on a compact domain and under periodic boundary conditions.

We turn next into investigating the FJ model under antiperiodic boundary conditions. In this case, the transformation (8) is not allowed because it does not preserve the boundary conditions [14]. Hence, the theory exhibits no symmetry and, correspondingly, first-class constraints should not arise. In other words, under antiperiodic boundary conditions, the FJ model is a pure secondclass system whose excitations are all chiral. To check that this is indeed the case, we go again into the discrete formulation.

Instead of (10) we write, in the present case,

$$
\begin{aligned}
\phi\left(x^{0}, x^{1}\right)= & \frac{1}{2 R} \sum_{n \geq 0}\left\{\left[a_{n}\left(x^{0}\right)+i b_{n}\left(x^{0}\right)\right] e^{i \omega_{n+\frac{1}{2}} x^{1}}\right. \\
& \left.+\left[a_{n}\left(x^{0}\right)-i b_{n}\left(x^{0}\right)\right] e^{-i \omega_{n+\frac{1}{2}} x^{1}}\right\}
\end{aligned}
$$

This time the canonical analysis reveals that all the constraints are, in fact, second class.

The system is quantized by using, once more, the Dirac-brackets quantization procedure. We omit the de- 
tails and just mention that, this time, the quantum dynamics is solved by the field operator

$$
\begin{aligned}
\hat{\phi}\left(x^{0}, x^{1}\right)= & \frac{1}{\sqrt{2 \pi}}\left(\frac{\pi}{R}\right) \sum_{n \geq 0} \frac{1}{\sqrt{\omega_{n+\frac{1}{2}}}}\left[\hat{\Lambda}_{n} e^{-i \omega_{n+\frac{1}{2}}\left(x^{0}+x^{1}\right)}\right. \\
& \left.+\hat{\Lambda}_{n}^{\dagger} e^{i \omega_{n+\frac{1}{2}}\left(x^{0}+x^{1}\right)}\right]
\end{aligned}
$$

where $\hat{\Lambda}_{n}$ and $\hat{\Lambda}_{n}^{\dagger}$ are, for all values of $n$, destruction and creation operators, respectively.

Therefore, the vacuum expectation value of $\hat{\phi}$ vanishes implying that, for antiperiodic boundary conditions, the vacuum is unique. We also remark that the chiral nature of $\hat{\phi}$ survives the limit $R \rightarrow \infty$ and that (4) is obeyed.

Since the field $\hat{\phi}$ is itself chiral, a symmetric quantum energy-momentum tensor can be constructed by using (22) with $\hat{\Phi}$ replaced by $\hat{\phi}$. As for periodic boundary conditions, the equal-time algebra of densities is a Virasoro algebra. Moreover, a set of charges can be constructed which act as generators of the Poincaré group.

To summarize, after recognizing the improper nature of the constraints, we were able to perform a consistent quantization of the FJ model as a constrained system. It became clear that each boundary condition defines a different problem, mainly in connection with the symmetry content of the theory: while the periodic case is a gauge theory, the antiperiodic is not. In both cases the physical excitations are all chiral.

Supported in part by Conselho Nacional de Desenvolvimento Científico e Tecnológico, CNPq, Brazil.
[1] D. J. Gross, J. A. Harvey, E. Martinec, and R. Rohm, Phys. Rev. Lett. 54, 502 (1985).

[2] X. G. Wen, Phys. Rev. Lett 64, 2206 (1987).

[3] P. P. Srivastava, Phys. Rev. Lett 63, 2791 (1989).

[4] D. S. Kulshreshtha and H. J. W. Müller-Kirsten, Phys. Rev. D 45, R393 (1992).

[5] H. O. Girotti, M. Gomes, and V. O. Rivelles, Phys. Rev D 45, R3329 (1992).

[6] W. Siegel, Nucl. Phys. B238, 307 (1984); M. Bernstein and J. Sonnenschein, Phys. Rev. Lett. 60, 1772 (1988); M. Henneaux and C. Teitelboim, Phys. Lett. B 206, 650 (1988); E. Abdalla, M. C. B. Abdalla, F. P. Devecchi, and A. Zadra, ibid. 215, 564 (1988).

[7] C. Imbimbo and A. Schwimmer, Phys. Lett. B 193, 455 (1987); J. M. Labastida and M. Pernici, Nucl. Phys. B297, 557 (1988); L. Mezincescu and R. Nepomechie, Phys. Rev. D 37, 3067 (1988).

[8] R. Floreanini and R. Jackiw, Phys. Rev. Lett. 59, 1873 (1987).

[9] Throughout this paper we use natural units $(c=\hbar=1)$. Our metric is $g_{00}=-g_{11}=1$.

[10] P. A. M. Dirac, Lectures on Quantum Mechanics (Belfer
Graduate School of Science, Yeshiva University Press, New York, 1964).

[11] M. E. V. Costa and H. O. Girotti, Phys. Rev. Lett. 60, 1771 (1988); also, see H. O. Girotti, M. Gomes, V. Kurak, V. O. Rivelles, and A. J. da Silva, ibid. 60, 1913 (1988).

[12] An alternative quantization scheme is possible in this case. See, in this connection, Ref. [8] and L. Faddeev and R. Jackiw, Phys. Rev. Lett. 59, 1873 (1987).

[13] J. Barcelos Neto and C. Wotzasek, Europhys. Lett. 21, 511 (1993).

[14] R. Benguria, P. Cordero, and C. Teitelboim, Nucl. Phys B122, 61 (1976).

[15] P. J. Steinhardt, Ann. Phys. (N.Y.) 128, 425 (1980).

[16] K. Sundermeyer, Constrained Dynamics (SpringerVerlag, Berlin, 1982).

[17] P. Srivastava, University of Padova Report No. DFPF/92/TH/58 (unpublished).

[18] A similar technique, but in connection with another model for chiral bosons, has been used by J. Reyes Martinez, A. H. Zimerman, and P. Teotonio Sobrinho, Phys. Rev. D 39, 3055 (1989).

[19] J. Schwinger, Phys. Rev. 127, 324 (1962). 